\title{
La formación de médicos y enfermeras del trabajo en España: una mirada histórica hasta llegar al presente
}

\author{
Training of occupational physicians and nurses in \\ Spain: a historical review
}

\author{
José María Ramada $1,2,3$ \\ Gloria Nohales ${ }^{4}$ \\ 'Unidad Docente Multidisciplinar de Salud Laboral Parc de Salut Mar, Barcelona, España \\ 2Institut Hospital del Mar d'Investigacions Mèdiques (IMIM), Barcelona, España \\ ${ }^{3}$ CIBER de Epidemiología y Salud Pública (CIBERESP), Madrid, España \\ ${ }^{4}$ Jefa de Estudios. Comisión de Docencia Parc de Salut Mar, Barcelona, España
}

Fechas · Dates

Recibido: 2019.11 .09

Aceptado: 2019.12.07

Publicado: 2020.01.15

\section{Correspondencia $\cdot$ Corresponding Author}

\section{José María Ramada}

E-mail: JRamada@parcdesalutmar.cat 
Con el título Quo Vadis Salud Laboral? se ha presentado este año la XXIX Diada de la Associació Catalana de Salut Laboral, que ha tenido lugar en el marco incomparable del recinto modernista del Hospital de Sant Pau de Barcelona, declarado Patrimonio Mundial por la UNESCO en 1997. A ningún profesional sanitario de la salud laboral le pareció extraña la cuestión planteada (Quo Vadis?) porque con los años, la incertidumbre sobre el futuro de las dos especialidades preventivas (sanitarias) de largo recorrido en España y además previstas en la Ley 31/1995 de Prevención de Riesgos Laborales se ha convertido ya en un problema secular.

La OMS define la medicina del trabajo como «la especialidad médica que, actuando aislada o comunitariamente, estudia los medios preventivos para conseguir el más alto grado de bienestar físico, psíquico y social de los trabajadores, en relación con la capacidad de éstos, con las características y riesgos de su trabajo, el ambiente laboral y la influencia de éste en su entorno, así como promueve los medios para el diagnóstico, tratamiento, adaptación, rehabilitación y calificación de la patología producida o condicionada por el trabajo». Pero tal como se explica en Ruiz-Frutos ${ }^{(1)}$, haciendo referencia a un artículo de Macdonald ${ }^{(2)}$, las competencias de los médicos del trabajo ya fueron claramente descritas por Bernardino Ramazzini en su obra De Morbis Artificum Diatriba (Tratado sobre las enfermedades de los trabajadores)(3), cuando enfatizaba en la importancia de que los médicos debían realizar una exhaustiva historia laboral reforzando la afirmación de Hipócrates de que todo médico está obligado a preguntar por la ocupación de sus pacientes.

A pesar de la relevancia clínica, preventiva y social que emana de la definición de la OMS, la medicina y enfermería del trabajo son disciplinas ausentes o escasamente presentes en los planes de formación de los grados de medicina y enfermería. Salvo honrosas excepciones, los médicos y las enfermeras acaban sus estudios de grado en las Facultades de Medicina y en las Escuelas Universitarias de Enfermería sin haber cursado siquiera una asignatura que les explique la evidencia científica existente sobre cómo abordar los problemas que se originan por las interacciones entre las condiciones de trabajo y la salud de las personas( ${ }^{(4)}$. Este no es un asunto baladí. Nuestros médicos y enfermeras, en su mayoría, al finalizar los estudios de grado adolecen de una formación curricular mínima sobre los fundamentos de la salud laboral y su impacto en la prevención, diagnóstico y tratamiento de las enfermedades ocasionadas por la exposición a factores de riesgo en el trabajo. Esta laguna curricular podría explicar el escaso interés de los profesionales sanitarios por las especialidades de medicina y enfermería del trabajo, que se ha venido reflejando en los sucesivos años con la elección de estas especialidades en los últimos puestos de las plazas ofertadas para la formación MIR y EIR.

Los antecedentes más recientes de la medicina del trabajo en España podríamos situarlos en el año 1956, cuando España adoptó la recomendación 112 de la OIT (sobre los servicios de medicina del trabo) ${ }^{(5)}$ mediante la publicación del Decreto por el que se organizaban los Servicios Médicos de Empresa (SME) ${ }^{(6)}$ y su desarrollo reglamentario en 1959(7). La regulación de la formación que debían recibir los 
médicos y enfermeras para permitirles el ejercicio de la medicina en los SME fue un hito importante en la historia de estas especialidades.

Inicialmente, esta formación tenía una duración aproximada de seis meses, se impartía en la Escuela Nacional de Medicina del Trabajo (que contaba con escuelas departamentales en diferentes provincias) y con la superación del mismo se obtenía el Diploma en Medicina de Empresa o de ATS de Empresa. En 1984 se publicó el Real Decreto 127/1984 que regulaba la formación médica especializada y la obtención del título de médico especialista (para todas las especialidades) ${ }^{(8)}$. A partir de este momento, la formación de especialistas en medicina del trabajo se impartiría en las Escuelas Profesionales de Medicina del Trabajo (EPMT) adscritas a diversas Universidades y en las que cada alumno de la especialidad financiaba su formación. Por un tiempo, hubo libre acceso a las EPMT fue de libre acceso y el periodo académico para la obtención del título de especialista era de dos años. En 1988 una Orden Ministerial(9) dio desarrollo normativo a los estudios de las especialidades del apartado 3 del RD 127/1984, incluyendo la especialidad de medicina del trabajo y requiriendo un periodo académico de tres años impartidos en las EPMT. A partir de este momento, el acceso a la especialidad se realizó por concurso de méritos durante unos años y posteriormente a través del examen MIR, aunque la financiación de estas plazas siguió siendo asumida por cada estudiante de la especialidad.

En Europa se produjeron iniciativas encaminadas a la integración y homogenización de la formación de los médicos del trabajo con el objetivo de armonizar su formación en todos los países. En 1997 tuvo lugar una conferencia en Glasgow en la que se promovió un estudio Delphi(2) en el que participaron miembros de sociedades profesionales como son la Occupational Medicine Section of the European Union of Medical Specialists (UEMS) ${ }^{(10)}$, la European Association of Schools of Occupational Medicine (EASOM) ${ }^{(11)}$ y la European Network of Societies of Occupational Physicians (ENSOP), con el objetivo de identificar los requerimientos para la formación de médicos del trabajo en Europa. Sin embargo, a pesar de que las recomendaciones han sido asumidas por todas estas sociedades, existen diferencias en la formación requerida en los países de la Unión Europea ${ }^{(12)}$. Asimismo, en España se produjeron iniciativas pioneras con el objetivo de definir las competencias profesionales de los médicos del trabajo, como la liderada por Serra ${ }^{(13)}$ del Centro de Investigación en Salud Laboral (CiSAL-UPF).

En 2005, una nueva Orden del Ministerio de Sanidad y Consumo(14) introdujo un profundo cambio en el modelo formativo de la especialidad. Los MIR de medicina del trabajo pasaron de una formación académica (impartida en las EPMT y financiada por los propios alumnos) a una formación que se realizaba en la Unidades Docentes de Medicina del Trabajo (UDMT), con una importante actualización del programa formativo, de cuatro años de duración, que incorporaba rotaciones en centros hospitalarios, de atención primaria y otras Unidades/Servicios/Institutos relacionados con la salud de los trabajadores y quedando esta especialidad integrada con el resto de especialidades médicas remuneradas del sistema de Médicos Internos Residentes (MIR). 
Sin embargo, desde el principio se estableció una diferencia relativa a la financiación de las plazas de éstos especialistas, no realizándose a través del Sistema Público de Salud (SPS), como el resto de especialidades médicas y de enfermería, sino a través de diversas entidades, con variaciones según cada CCAA, incluyendo a las Mutuas de Accidentes de Trabajo y Enfermedades Profesionales (actualmente, Mutuas Colaboradoras con la Seguridad Social, en adelante Mutuas), Consejerías de las CCAA, Servicios de Prevención Privados o Centros Públicos especializados.

Este mismo año (2005) se produjo el reconocimiento de la enfermería del trabajo como especialidad de enfermería(15). Tomando como base iniciativas previas existentes para la definición de las funciones y tareas nucleares de las enfermeras del trabajo(16), tras su reconocimiento como especialidad de enfermería, Labarta(17) (en nombre del Grupo de trabajo «Enfermería del trabajo» de la Societat Catalana de Seguretat i Medicina del Treball) publicó una propuesta sobre las competencias de éstas. En 2008 se publica un Real Decreto(18) en el que se crean las Unidades Docentes Multiprofesionales, entre ellas las de salud laboral, para la formación de especialistas en medicina y enfermería del trabajo. El programa formativo de la especialidad de enfermería del trabajo no quedaría regulado en el Boletín Oficial del Estado hasta el año 2009(19) requiriéndose un periodo formativo de dos años e integrándose con el resto de especialidades de enfermería en el sistema de Enfermeras Internas Residentes (EIR) y con un sistema de financiación idéntico al de los MIR de medicina del trabajo.

Este hecho diferencial, relacionado con las fuentes de financiación de las plazas ofertadas para médicos y enfermeras del trabajo por la vía MIR y EIR, ha influido significativamente en la dramática reducción del número de plazas ofertadas para la formación de estos especialistas en los últimos años. Se estima que cuando la formación de los especialistas en medicina del trabajo se realizaba en las Escuelas Profesionales Universitarias, y las plazas ofertadas eran financiadas por los propios estudiantes de la especialidad, se convocaban unas 300 plazas anuales. Sin embargo, en el año 2005, con la entrada en vigor del sistema MIR se ofertaron 77 plazas de medicina del trabajo para toda España y, de un total de 159 plazas acreditadas, el máximo histórico se produjo en el año 2009 con una oferta de 135 plazas (Figura 1), no llegando sin embargo a saturarse nunca la capacidad formativa acreditada.

A partir de este año (2009), con la llegada de la crisis económica, las plazas ofertadas por las Mutuas y otras entidades privadas se fueron reduciendo progresivamente, hasta llegar al mínimo histórico de 45 plazas ofertadas en 2015 para toda España. En CCAA con plazas acreditadas para la formación MIR de medicina del trabajo en las que la financiación provenía en su inmensa mayoría de las Mutuas por primera vez no se ofertó ninguna plaza en 2013 (Aragón, Castilla-La Mancha y Cataluña), y en los años sucesivos se sumaron a esta dramática situación las CCAA de Baleares, Ceuta y La Rioja.

En Cataluña, en el periodo 2013-2015 no se ofertó ninguna plaza MIR de medicina del trabajo (Figura 1). En 2016, se ofertó una plaza (única para toda Cataluña) fi- 
nanciada por el Parc de Salut Mar (PSMAR), institución sanitaria del SPS. La plaza está adscrita a la UDMT «Mateu Orfila» de la Universidad Pompeu Fabra y desde entonces se ha venido ofertando cada año como única plaza MIR de medicina del trabajo y desde 2018 junto a la Corporació Parc Taulí de Sabadell que oferta hasta ahora una segunda plaza, todas financiadas por el Sistema Nacional de Salud.

En 2019 el PSMAR ha acreditado, tras años de haber iniciado el proceso, la primera Unidad Docente Multiprofesional de Salud Laboral en Cataluña (UDMSL-PSMAR) para la formación de médicos y enfermeras del trabajo, adscrita a la Comisión de Docencia de esta Corporación Sanitaria de titularidad pública. La acreditación se ha realizado con una capacidad docente para la formación de 2 MIR de medicina del trabajo y 2 EIR de enfermería del trabajo, ofertando para el año 2020 una plaza MIR y dos plazas EIR.

La experiencia de estos años nos ha enseñado que el único sistema de financiación que ha garantizado mínimamente la regularidad en el número de plazas ofertadas para la formación de médicos y enfermeras especialistas ha sido el SPS. Los vaivenes de la situación económica no han tenido tanto impacto en esta vía de financiación y por tanto es posible que el modelo de futuro para la formación de médicos y enfermeras del trabajo pase por la acreditación de Unidades Docentes Multiprofesionales de Salud Laboral en los Hospitales de titularidad pública. Ello equipararía definitivamente los modelos formativos de las especialidades de medicina y enfermería del trabajo con el resto de especialidades médicas y de enfermería a las que se accede por la vía MIR/EIR.

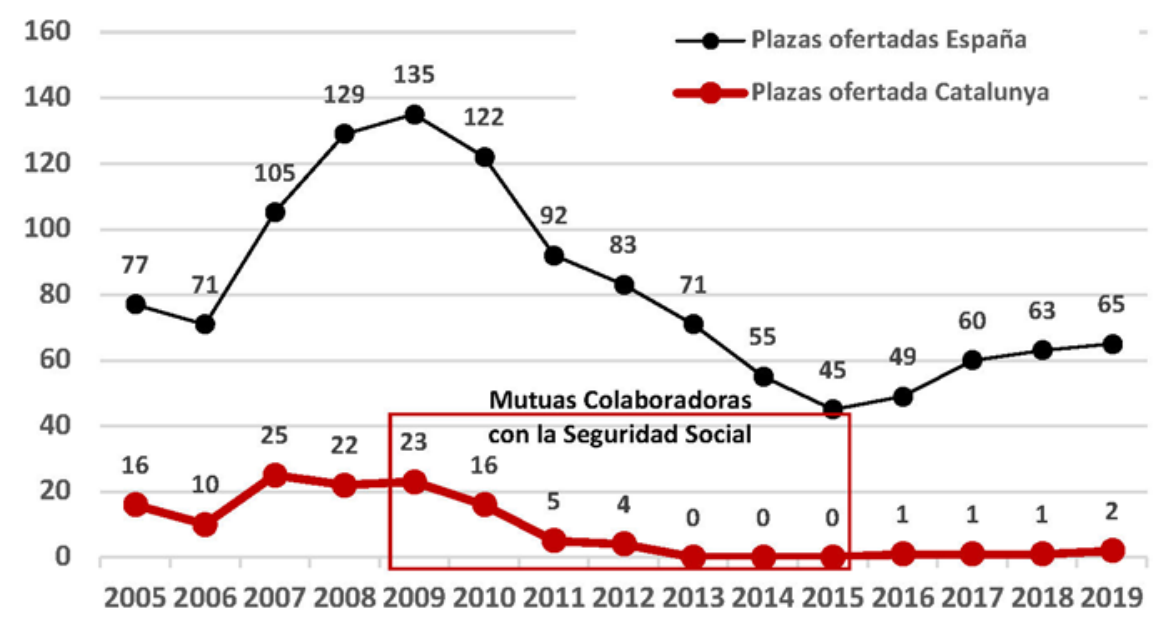

Figura 1: Número de plazas MIR ofertadas en España y en Cataluña (año incorporación R1). Periodo 2005-2019. Fuente: elaboración propia, a partir de las Convocatorias anuales. Boletín Oficial del Estado (BOE).

La convocatoria 2020 de plazas MIR de medicina del trabajo oferta 94 plazas y la de EIR de enfermería del trabajo 62, aproximándose por primera vez al número de plazas ofertadas de medicina del trabajo, lo que pone en evidencia la insuficiencia 
de la oferta de plazas EIR de enfermería del trabajo en las convocatorias de los años anteriores (Figura 2).

\section{PLAZAS EIR OFERTADAS}

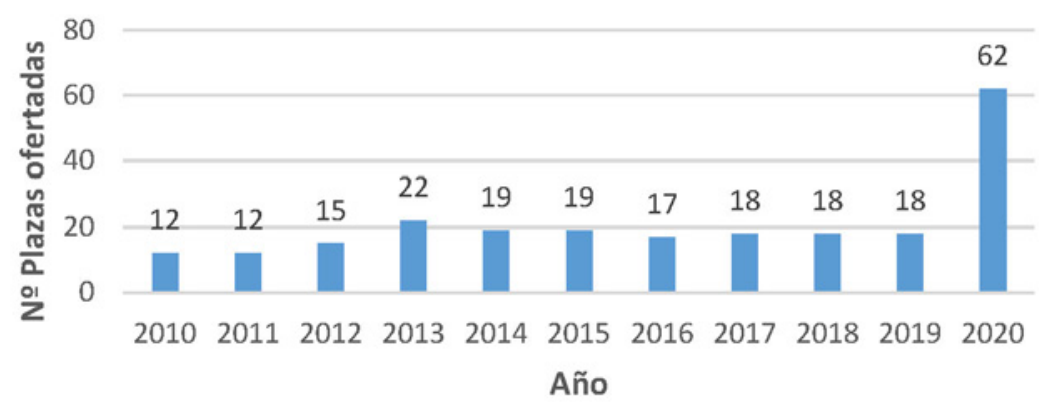

Figura 2: Número de plazas EIR ofertadas en España. Periodo 2010-2020. Fuente: elaboración propia, a partir de las Convocatorias anuales. Boletín Oficial del Estado (BOE).

Actualmente, en España no existe un registro de profesionales sanitarios que ofrezca una información precisa sobre el número y características sociodemográficas de los profesionales de cada especialidad. Ello dificulta un cálculo ajustado de las necesidades de las diferentes especialidades médicas y de enfermería. Sin embargo, el estudio sobre demografía médica publicado en 2018 por el Consejo General de Colegios de Médicos ${ }^{(20)}$ describe que los especialistas en medicina del trabajo son la tercera población más envejecida dentro de las diferentes especialidades médicas (después de estomatología y análisis clínicos), con un promedio de edad de 55 años (un 62,4\% > 55 años) y una ratio de reposición del 0,2\% (número de especialistas que repondrán a los que se jubilen en los próximos 10 años), siendo la más baja de todas las especialidades médicas. Asimismo, detalla que mientras en Europa la densidad de médicos del trabajo es en promedio de 6,4 médicos del trabajo por cada 100.000 habitantes (EUROSTAT, 2015) en España es de 2,9. Datos similares se ofrecen en el informe publicado en la página web del Ministerio de Sanidad, Consumo y Bienestar Social en 2019 sobre la estimación de la oferta y demanda de médicos especialistas para el periodo 2018-2030(21). En este informe se describe una población de especialistas en medicina del trabajo envejecida, con unas tasas de reposición procedentes de la vía MIR muy bajas y con una variabilidad del número de especialistas muy alta dependiendo de cada CCAA.

Así pues, en el momento actual se conjugan varias situaciones que hay que resolver. Tenemos una población trabajadora que tiene derecho a que se le proteja de los riesgos del trabajo y a no enfermar como consecuencia del mismo. Tenemos una Administración Pública que no ha sabido o no ha tenido la voluntad política suficiente para dar una respuesta adecuada a las necesidades existentes de médicos y enfermeras del trabajo. Y tenemos a los colectivos profesionales desorientados por la existencia de un modelo de actuación en crisis, derivado del rol que actualmente están jugando en los Servicios de Prevención. 
Urge que las Administraciones (Autonómicas y del Estado) adopten medidas que corrijan la situación actual. La lentitud administrativa en el proceso de acreditación de Unidades Docentes Multiprofesionales ha sido insoportable hasta hace muy poco. Urge que las Consejerías de Salud/Sanidad de las CCAA, con competencias en el SPS, se decidan definitivamente a financiar de manera estable un número de plazas MIR y EIR que responda a las necesidades reales de la población trabajadora de cada CCAA. Urge que las sociedades científicas profesionales ejerzan con más entusiasmo y liderazgo su capacidad de influir. ¿Para cuándo los documentos de posicionamiento?, ¿Para cuándo una carta a la Conferencia Nacional de Decanos de Enfermería y de Facultades de Medicina Españolas, explicando las carencias curriculares en salud laboral de los graduados en medicina y enfermería?

Y finalmente, mientras esto sucede, urge que los profesionales de la medicina y enfermería del trabajo se cuestionen la utilidad del enfoque victimista que tan presente ha estado en los últimos años en los debates sobre el futuro de estas especialidades. Seguro que la reivindicación es lícita y necesaria, pero además se hace ineludible una reflexión sobre cómo, desde la profesión, los especialistas españoles en medicina y enfermería del trabajo están haciendo avanzar el conocimiento científico de la especialidad. Las universidades, los hospitales y las especialidades sanitarias que investigan poco, suelen tener poco reconocimiento social y encuentran mayores dificultades para convertirse en una prioridad a la que destinar recursos públicos. Urgen especialistas que desarrollen tesis doctorales, que realicen investigación científica de calidad en salud laboral y publiquen en revistas científicas de prestigio, y esto en gran medida depende de la decisión personal de cada especialista en activo, eso sí, en un entorno que lo facilite y promueva.

\section{Bibliografía}

1. Ruiz-Frutos C, García AM, Benavides F. Especialidades en salud laboral. En: Ruiz-Frutos C, García AM, Delclos J, Benavides F, coordinadores. Salud laboral. Conceptos y técnicas para la prevención de riesgos laborales. $3^{\mathrm{a}}$ ed. Barcelona: Masson; 2007. p. 62-63.

2. Macdonald EB, Ritchie KA, Murray KJ, Gilmour WH. Requirements for occupational medicine training in Europe: a Delphi study. Occup Environ Med. 2000; 57: 98-105.

3. Ramazzini B (autor), Araujo-Alvarez JM (traductor). Las enfermedades de los trabajadores: De Morbis Artificum Diatriba. $1^{\text {a }}$ ed. México, D.F.: Miguel Ángel Porrúa/UAM; 2008.

4. López AA, Ayensa JA, Núñez C, Vicente-Herrero MT. Asignaturas específicas o relacionades con la Medicina del Trabajo en las facultades de Medicina españolas. Arch Prev Riesgos Labor. 2007; 10: 188-19.

5. Decreto de 21 de agosto de 1956 por el que se organizan los Servicios Médicos de Empresa. Boletín Oficial del Estado, n² 287, (13-10-1956). 
6. Organización Internacional del Trabajo (OIT) [sede Web]. R112 - Recomendación sobre los servicios de medicina del trabajo. [consultado el 30 de noviembre de 2019]. Disponible en: https://www.lo.org/dyn/normlex/es/f?p=NORMLEXPUB:12100:0::NO:12100:P12100_INSTRUMENT_ID:312450:NO

7. Orden de 21 de noviembre de 1959 por la que se aprueba el Reglamento de los Servicios Médicos de Empresa. Boletín Oficial del Estado, n² 284, (27-11-1959).

8. Real Decreto 127/1984, de 11 de enero, por el que se regula la formación médica especializada y la obtención del título de Médico Especialista. Boletín Oficial del Estado, n²6, (31-1-1984).

9. Orden de 9 de septiembre de 1988 de acceso a las especialidades del apartado 3 del anexo del Real Decreto 127/1984, de 11 de enero. Boletín Oficial del Estado, n०219, (12-09-1988).

10. European Union of Medical Specialists (UEMS) [sede Web]. Bruselas; 2019, [actualizada el 19 de octubre de 2019; consultado el 21 de noviembre de 2019]. Disponible en: https://www.uems.eu/

11. European Association of Schools of Occupational Medicine (EASOM) [sede Web]. Luxemburgo; 2019, [actualizada agosto de 2019; consultado el 21 de noviembre de 2019]. Disponible en: https://www.easom.eu/

12. Macdonald E, Baranski B, Wilford J. Occupational Medicine in Europe: Scope and Competencies. Health, Environment and Safety in Enterprises Series n ${ }^{\circ} 3$. WHO European Centre for Environment and Health. Bithoven, 2000 [consultado el 21 de noviembre de 2019]. Disponible en: https://www.who.int/occupational_health/regions/en/oeheuroccmedicine.pdf

13. Serra C, Brotons A, Plana M, Benavides FG, Maqueda J, en nombre del Grupo de Trabajo sobre las competencias profesionales de los médicos del trabajo. Las competencias profesionales de los médicos del trabajo. Barcelona: Mutual Cyclops, 2003.

14. Orden SCO/1526/2005, de 5 de mayo, por la que se aprueba y publica el programa formativo de la especialidad de Medicina del Trabajo. Boletín Oficial del Estado, no 127, (28-05-2005).

15. Real Decreto 450/2005, de 22 de abril, sobre especialidades de Enfermería. Boletín Oficial del Estado, no 108, (06-05-2005).

16. Grupo de Trabajo sobre Enfermería del Trabajo. Funciones, actividades y tareas nucleares de los diplomados universitarios de enfermería especialistas en enfermería del trabajo. Arch Prev Riesgos Labor. 2002;5:30-1.

17. Labarta RM, López MV, Plana M. (en nombre del Grupo de trabajo «Enfermería del trabajo» de la Societat Catalana de Seguretat i Medicina del Treball). Las competencias profesionales de la enfermería del trabajo. Arch Prev Riesgos Labor. 2006; 9: 89-93. 
18. Real Decreto 183/2008, de 8 de febrero, por el que se determinan y clasifican las especialidades en Ciencias de la Salud y se desarrollan determinados aspectos del sistema de formación sanitaria especializada. Boletín Oficial del Estado, $n^{\circ} 45$, (21-8-2008).

19. Orden SAS/1348/2009, de 6 de mayo, por la que se aprueba y publica el programa formativo de la especialidad de Enfermería del Trabajo. Boletín Oficial del Estado, no 129, (28-05-2009).

20. Consejo General de Colegios de Médicos (Cuadernos CGCOM) [sede Web]. Estudio sobre demografía Médica, 1018. [consultado el 27 de noviembre de 2019]. Disponible en: https://www.cgcom.es/sites/default/files/estudio_demografia_2017/

21. Ministerio de Sanidad, Consumo y Bienestar Social. Barber-Perez P, Gonzalez López-Valcarcer B. Estimación de la oferta y demanda de médicos especialistas. España 2018-2030. [consultado el 27 de noviembre de 2019]. Disponible en: https://www.mscbs.gob.es/profesionales/formacion/necesidadEspecialistas/doc/20182030EstimacionOfertaDemandaMedicosEspecialistasV2.pdf 